\title{
ON THE INTERPRETATION OF THE CHARM II DATA
}

\author{
V.A. Novikov \\ ITEP, Moscow, 117259, Russia \\ L.B. Okun ${ }^{*)}$ \\ Theoretical Physics Division, CERN \\ CH - 1211 Geneva 23 \\ and \\ M.I. Vysotsky \\ ITEP, Moscow, 117259, Russia
}

\begin{abstract}
The data of the CHARM II and of the ALEPH, DELPHI, L3 and OPAL Collaborations are compared in the framework of $\left(\alpha\left(m_{Z}\right), m_{Z}, G_{\mu}, m_{t}, m_{H}\right)$-parametrization of the electroweak radiative corrections. The comparison shows that the physical meaning of the CHARM II results is more significant than was stated in the original publication of the CHARM Collaboration. In particular, CHARM II is very sensitive to the $Z$ boson coupling to the muon neutrinos.
\end{abstract}

*) Permanent address: ITEP, Moscow, 117259, Russia.

CERN-TH.6695/92

October 1992 
Great experimental and theoretical efforts have been devoted during recent years to the precision tests of the Electroweak Minimal Standard Model. These tests would be unthinkable without elaborated computer codes, in particular those which implement electroweak radiative corrections. However, the use of computer codes cannot devaluate the traditional "old-fashioned" theoretical approach, based on the comparison of experimental data with the explicit analytic expressions in the framework of rational parametrization. In this note we apply such an approach to the new CHARM II data [1] (see also [2]).

As was shown in our papers [3,4], all electroweak precision data are, within $1 \sigma$, described by the Born amplitudes using as input parameters

$$
\begin{gathered}
\bar{\alpha}=\alpha\left(m_{Z}\right)=1 / 128.8(1) \quad[5] \\
m_{Z}=91.187(7) \mathrm{GeV} \quad[6] \\
G_{\mu}=1.16639(2) \times 10^{-5} \mathrm{GeV}^{-2} \quad[7]
\end{gathered}
$$

The remaining, small, genuinely electroweak corrections were explicitly expressed in [4] for leptonic $Z$-decays in terms of elementary functions of unknown masses of the top-quark, $m_{t}$, and the Higgs boson, $m_{H}$, and a very accurately known angle $\theta$, introduced by Peskin [8]:

$$
\begin{gathered}
4 \sin ^{2} \theta \cos ^{2} \theta=4 c^{2} s^{2}=\sin ^{2} 2 \theta=\frac{4 \pi \bar{\alpha}}{\sqrt{2} G_{\mu} m_{Z}^{2}}=0.7113(6) \\
\theta=0.50185(37), s^{2}=0.2314(3) .
\end{gathered}
$$

The observables of leptonic $Z$-decays are:

$$
\begin{gathered}
\Gamma_{\nu_{\mu}}=\frac{\sqrt{2} G_{\mu} m_{Z}^{3}}{12 \pi} \cdot\left(g^{\nu_{\mu}}\right)^{2}, \\
g^{\nu_{\mu}}=\frac{1}{2}+\frac{3 \bar{\alpha} V_{\nu_{\mu}}(t, h)}{64 \pi s^{2} c^{2}}, \\
\Gamma_{e}=\frac{\sqrt{2} G_{\mu} m_{Z}^{3}}{12}\left(1+\frac{3 \bar{\alpha}}{4 \pi}\right)\left[\left(g_{A}^{e}\right)^{2}+\left(g_{V}^{e}\right)^{2}\right], \\
g_{A}^{e}=-\frac{1}{2}-\frac{3 \bar{\alpha} V_{A}(t, h)}{64 \pi s^{2} c^{2}}, \\
R=g_{V}^{e} / g_{A}^{e}=1+\left(1-4 s^{2}\right)+\frac{3 \bar{\alpha}}{4 \pi\left(c^{2}-s^{2}\right)} V_{R}(t, h),
\end{gathered}
$$

where $t=\left(m_{t} / m_{Z}\right)^{2}, \quad h=\left(m_{H} / m_{Z}\right)^{2}$, while functions $V_{\nu_{\mu}}, V_{A}$, and $V_{R}$ are given respectively by Eqs. (70), (97) and (111) of ref. [4]. They are chosen in such a way that $V \simeq t$ for $t \gg 1[9]$.

Let us now consider the elastic scattering $\nu_{\mu} e \rightarrow \nu_{\mu} e$. The matrix element of this process in given by $[1,2,3]$ :

$$
M_{\nu_{\mu} e}=\frac{G}{\sqrt{2}}\left[\bar{\nu} \gamma_{\alpha}\left(1+\gamma_{5}\right) \nu\right]\left[\bar{e}\left(g_{A}^{e \nu_{\mu}} \gamma_{\alpha} \gamma_{5}+g_{V}^{e \nu_{\mu}} \gamma_{\alpha}\right) e\right]
$$

where according to [1]:

$$
g_{A}^{e \nu_{\mu}}=-0.503 \pm 0.018
$$




$$
g_{V}^{e \nu_{\mu}}=-0.025 \pm 0.019
$$

What is the theoretical relation between the constants $g_{A, V}^{e \nu_{\mu}}$ on the one hand, and $g_{A, V}^{e}$ and $g^{\nu_{\mu}}$ on the other?

First of all, in spite of the fact that the former are measured at $q^{2} \simeq-0.1 \mathrm{GeV}^{2}$, while the latter at $q^{2} \simeq 10^{4} \mathrm{GeV}^{2}$, they both belong to the scale $q^{2} \simeq m_{W . Z}^{2}$. The point is that the couplings of the $W$ - and $Z$-bosons, $\alpha_{W}$ and $\alpha_{Z}$, do not run unless $\left|q^{2}\right| \gg m_{W, Z}^{2}$. There are two necessary conditions for the running of a gauge coupling constant: 1 ) the particles in the vacuum polarization loops must be light, 2) the gauge particles themselves must be light (light means $m^{2} \ll\left|q^{2}\right|$ ). The first condition is fulfilled in our case, but the second is not. This explains why the natural parameter which enters all electroweak physics is $\bar{\alpha} \equiv \alpha\left(m_{Z}\right)$, but not $\alpha=\alpha(0)$. [It is $\alpha(0)$ that ran away from the natural electroweak value $\bar{\alpha}=\alpha\left(m_{Z}\right)$.

Secondly, in spite of similar notations, $g_{A, V}^{e \nu}$ on the one hand and $g_{A, V}^{e}\left(g^{\nu}\right)$ on the other hand have different physical meaning. In fact if one neglects the box diagrams, the $\gamma Z$-mixing, and the neutrino charge radius in $\nu_{\mu} e$-scattering [which can be safely done with present experimental uncertainties in $g_{A}^{e \nu}$ and $g_{V}^{e \nu}$ (see below)], one can write, neglecting terms $O\left(\frac{\alpha}{\pi}\right)$ but keeping terms $O\left(\frac{\alpha}{\pi} t\right)$, simple factorization equations

$$
\begin{gathered}
g_{A}^{e \nu}=2 g^{\nu} g_{A}^{e} \\
g_{V}^{e \nu}=2 g^{\nu} g_{V}^{e} .
\end{gathered}
$$

This means that $g_{A, V}^{e \nu}$ should be compared not with $g_{A, V}^{e}$, as was done in ref. [1], but with products of couplings of electron and neutrino decays of $Z$-bosons.

According to ref. [6]:

$$
N_{\nu}=2.97(5)[\mathrm{A}], 3.10(6)[\mathrm{D}], 3.06(8)[\mathrm{L}], 3.06(8)[\mathrm{O}]
$$

(where A, D, L, O stands for ALEPH, DELPHI, L3, OPAL), which means, assuming universality of neutrino couplings to the $Z$-boson, that

$$
4\left(g^{\nu}\right)^{2} \simeq 0.990(17)[\mathrm{A}], 1.033(20)[\mathrm{D}], 1.020(27)[\mathrm{L}], 1.020(27)[\mathrm{O}] .
$$

The experimental accuracy for charged leptons is much better. Assuming lepton universality, one finds [10]:

$$
4\left(g_{A}^{l}\right)^{2}=1.006(6)[\mathrm{A}], 0.996(8)[\mathrm{D}], 0.996(6)[\mathrm{L}], 1.000(6)[\mathrm{O}],
$$

The square roots of products of corresponding numbers in (13) and (14) should be compared, according to (10), with [see eq. (8)]:

$$
\left|2 g_{A}^{e \nu_{\mu}}\right|=1.006 \pm 0.038 \text {. }
$$

Thus, the uncertainties in LEP experiments are smaller by a factor of $\sim 4$ for $\mathrm{A}$ and $\mathrm{D}$ and by a factor of 3 for $\mathrm{L}$ and $\mathrm{O}$. [If instead of $g_{A}^{l}$, we would consider $g_{A}^{e}$, the situation would be only slightly more favourable for CHARM II. Indeed, according to ref. [11], $\Gamma_{l^{+} l^{-}}=83.24(42) \mathrm{MeV}$, while $\left.\Gamma_{e^{+} e^{-}}=83.20(55) \mathrm{MeV}.\right]$ 
The unique importance of the CHARM II results is determined by the fact that their interpretation does not imply neutrino flavour universality. One cannot in principle (!) extract the $g^{\nu_{\mu}}$ from ADLO data.

Now let us turn from $g_{A}$ to $g_{V}$.

It follows from eqs. (10) and (11) that

$$
\frac{g_{V}^{e \nu}}{g_{A}^{e \nu}}=\frac{g_{V}^{e}}{g_{A}^{e}}
$$

From (8) and (9) we get

$$
g_{V}^{e \nu} / g_{A}^{e \nu}=0.050 \pm 0.038
$$

If we compare (17) with the combined LEP result, which assumes lepton universality $[6]:$

$$
g_{V}^{e} / g_{A}^{e}=1-4(0.2324(11))=0.0704(44),
$$

then it is obvious that the LEP accuracy is 8 times higher. However, for the decay $Z \rightarrow$ $e^{+} e^{-}$, the forward-backward asymmetry, which gives $g_{V}^{e} / g_{A}^{e}$ without any assumptions, is known with much lower accuracy. According to ref. [6], the values of $A_{F B}^{e}$ for the four LEP detectors are [L. Rolandi, private communication]:

$$
0.014(9)[\mathrm{A}], 0.013(13)[\mathrm{D}], 0.017(14)[\mathrm{L}],-0.002(12)[\mathrm{O}] .
$$

So only the ALEPH result differs from zero by $1.5 \sigma$. Each of these detectors has a rather low accuracy in measuring $A_{F B}^{e}$. However, the combined value for $g_{V}^{e} / g_{A}^{e}$ as derived from the equation

$$
A_{F B}^{e}=\frac{3\left(g_{A}^{e}\right)^{2}\left(g_{V}^{e}\right)^{2}}{\left(\left(g_{A}^{e}\right)^{2}+\left(g_{V}^{e}\right)^{2}\right)^{2}}
$$

is much more accurate:

$$
g_{V}^{e} / g_{A}^{e}=0.058_{-0.014}^{+0.020}
$$

Finally, we would like to make a theoretical remark concerning the neutrino charge radius caused by the vertex three-point loops, eeW, eWW, and the two-point loops, $e W$, in the neutrino "legs". One may also consider as a contribution to the neutrino charge radius the $Z$-pole diagram with a $Z \gamma$ transition. Both of them have light particles inside the loops and a massless particle (photon) outside. Therefore they "run", that is, they contain large logarithms of the type $\ln \left(m_{W}^{2} /\left|q^{2}\right|\right)$ or $\ln \left(m_{Z}^{2} /\left|q^{2}\right|\right)$ and $\ln \left(m_{Z}^{2} / m_{f}^{2}\right)$, where $f=\tau, u, d, s, c, b$, while $m_{f}$ is the corresponding mass (constituent for quarks: $\left.m_{u} \simeq m_{d}=0.4 \mathrm{GeV}, m_{s} \simeq 0.5 \mathrm{GeV}, m_{c}=1.5 \mathrm{GeV}, m_{b}=4.5 \mathrm{GeV}\right)$.

Both the vertex and the pole term give a contribution to $g_{V}^{e \nu}$. Fortunately, as was explained in detail in ref. [12], [13], [3], the logarithmic contribution of the vertex

$$
-\frac{\bar{\alpha}}{3 \pi} \ln \left|\frac{m_{W}^{2}}{\left|q^{2}\right|}\right| \simeq-6 \frac{\alpha}{3 \pi}
$$

is practically cancelled by the logarithmic contribution of the $Z \gamma$-transition:

$$
2 \frac{\bar{\alpha}}{3 \pi}\left\{\left(\frac{1}{4}-s^{2}\right)\left[2 \ln \left(\frac{m_{Z}}{|q|}\right)+\ln \left(\frac{m_{Z}}{m_{\tau}}\right)-\frac{5}{6}\right]+\right.
$$




$$
\begin{aligned}
& +\left(\frac{1}{4}-\frac{s^{2}}{3}\right)\left[\ln \left(\frac{m_{Z}}{m_{d}}\right)+\ln \left(\frac{m_{Z}}{m_{s}}\right)+\ln \left(\frac{m_{Z}}{m_{b}}\right)-\frac{5}{2}\right]+ \\
& \left.+\left(\frac{1}{2}-\frac{4}{3} s^{2}\right)\left[\ln \left(\frac{m_{Z}}{m_{u}}\right)+\ln \frac{m_{Z}}{m_{c}}-\frac{10}{6}\right]\right\} \simeq+7 \frac{\bar{\alpha}}{3 \pi}
\end{aligned}
$$

In conclusion, we have shown that the CHARM II experiment gives uniquely clean data for testing the lepton universality of the $Z$-boson interactions.

We are grateful to V. Khovansky and L. Maiani for stimulating discussions, and L. Rolandi for a helpful e-mail exchange.

\section{References}

[1] CHARM II Collaboration, Phys. Lett. B 281 (1992) 159.

[2] CHARM II Collaboration, Phys. Lett. B 259 (1991) 499.

[3] V. Novikov, L. Okun and M. Vysotsky, CERN-TH.6053/91, TPI-MINN-91/145 (1991).

[4] V. Novikov, L. Okun and M. Vysotsky, CERN-TH.6538/92, ITEP-67/92 (1992)

[5] H. Burkhardt, F. Jegerlehner, G. Penso and C. Verzegnassi, Z. Phys. C43 (1989) 497.

[6] L. Rolandi, Plenary talk given at the XXIst Int. Conf. on High Energy Physics, Dallas, Aug. 1992.

[7] Review of Particle Properties, Phys. Rev. D45, No. 11, part II (1992).

[8] M. Peskin, Lectures presented at the 17th SLAC Summer Institute on Physics at the $100 \mathrm{GeV}$ mass scale, 1989 (SLAC Summer Inst., Stanford, 1989), p. 71.

[9] M. Veltman, Nucl. Phys. B123 (1977) 89.

[10] D. Schaile, in: Les Rencontres de Physique de la Vallée d'Aoste, March 1992, Ed. M.Greco (Editions Frontières, Gif-sur-Yvette, 1992), in press.

[11] The LEP Collaborations: ALEPH, DELPHI, L3 and OPAL, Phys. Lett. B276 (1992) 247.

[12] F. Antonelli and L. Maiani, Nucl. Phys. B186 (1981) 269.

[13] S. Bellucci, M. Lusignoli and L. Maiani, Nucl. Phys. B189 (1981) 329. 